\title{
Control del movimiento de una silla de ruedas mediante sensor neuronal Emotiv
}

\section{Control of the movement of a wheelchair using an Emotiv neural sensor}

Guillermo David Amarilla Brassel ${ }^{1 *}$, Lorena Zalazar Benítez ${ }^{1}$, Norma Graciela Silva Ortiz ${ }^{1}$, ${ }^{1}$ Universidad Nacional de Asunción. Facultad Politécnica. Grupo de Investigación en Electrónica y Mecatrónica. San Lorenzo, Paraguay.*Autor de correspondencia: giem@pol.una.com.

Amarilla Brassel, G. D., Zalazar Benítez, L. \& Silva Ortiz, N. G. (2020). Control del movimiento de una silla de ruedas mediante sensor neuronal Emotiv. Revista Investigaciones y Estudios-UNA, 11(1), 2633. https://doi.org/10.47133/IEUNA4b

Resumen. Con el nuevo método de control del movimiento de una silla de ruedas mediante sensor neuronal se buscó obtener una tecnología mediante el cual se pueda ayudar a la interacción de personas con discapacidad motriz y su medio, teniendo como único canal de comunicación la actividad cerebral. El objetivo principal fue desarrollar un sistema que controle el movimiento de una silla de ruedas mediante sensor neuronal EMOTIV.

Mediante investigaciones, análisis y pruebas se obtuvo un índice de efectividad mayor al $75 \%$ para los estímulos de modo expresivo y $65 \%$ para los cognitivos para el control de la silla, teniendo en cuenta que para el uso de diferentes usuarios dichos controles requieren de entrenamiento previo para una mejor respuesta del sensor a los estímulos realizados.

Se logró la ejecución del sistema que permite que un usuario pueda controlar y manejar la silla mediante señales eléctricas producidas por el cerebro, de manera efectiva.

\begin{abstract}
With the new method of controlling the movement of a wheelchair by means of a neuronal sensor, it sought to obtain a technology in which the interaction of people with motor disabilities and their environment can be helped, having as only communication channel the cerebral activity. The main objective is to develop a system that controls the movement of a wheelchair using a neural sensor. The investigations by means of the analysis and the tests were obtained an index of the effectiveness the mayor $75 \%$ for the stimuli of expressive and $65 \%$ for the cognitive ones for the control of the chair, taking into account that for the use of diverse Prior to a better response of the sensor to the stimuli performed. The execution of the system was achieved allowing a user to control and manage the chair using electrical signals produced by the brain, effectively.
\end{abstract}

Keywords: control, emotiv, wheelchair.

Palabras clave: control, emotiv, silla de ruedas. 


\section{INTRODUCCIÓN}

La tetraplejía es una enfermedad de alto impacto psicosocial en los pacientes que la padecen y a sus allegados, dado por el alto grado de discapacidad que genera, produce una alta dependencia del paciente hacia la persona que lo cuida. De acuerdo con la Organización Mundial de la Salud, en 2010 cerca del $15 \%$ de la población mundial vivía con algún tipo de discapacidad, lo cual significa que alrededor de 1.000 millones de personas se encontraban en esta situación (World Health Organization, 2011). Para este mismo año en Estados Unidos, cerca de 265.000 personas habían sufrido lesión de espina dorsal de las cuales, cerca del $55 \%$ presentaban algún tipo de tetraplejía (National Spinal Cord Injury Statistical Center, 2011).

Gracias a los avances de la tecnología y a los desarrollos de la neurología, es posible la creación de ayudas tecnológicas que permitan mejorar la calidad de vida de pacientes con un alto grado de discapacidad, especialmente dirigidas a pacientes con tetraplejía. Una de las herramientas que ha surgido como una esperanza para mejorar la situación de estos pacientes, son las interfaces cerebrocomputador (BCI); las cuales son dispositivos que pueden leer las señales eléctricas del cerebro humano y traducirlas en señales de control para un computador (Wang, Esfahani \& Sundararajan, 2012). Éstas han presentado un gran desarrollo en los últimos años y han sido utilizadas en múltiples proyectos, algunos de ellos con fines médicos (Ang et al, 2011) y otros particularmente enfocados a personas con discapacidad severa. Desde principios de los años 90 se han realizado cirugías para implantar sensores neuronales que permiten transformar un pensamiento en una orden a un aparato electrónico. Con tales aditamentos una persona con limitación de movimientos puede controlar aparatos electrodomésticos y sillas motorizadas, o mover un brazo robot. Las señales cerebrales son adquiridas mediante un electroencefalograma (EEG) comercial llamado Emotiv Epoc el cual consta de 14 electrodos activos y 2 de referencia (Emotiv, 2017).

Dichas señales son filtradas y amplificadas en el EEG, y enviadas inalámbricamente hacia una PC portátil. El EEG Emotiv Epoc es capaz de detectar gesticulaciones faciales, estados emocionales y permite entrenar diversos comandos mentales.

Aprovechando este campo de investigación el objetivo principal de este proyecto fue el desarrollo de un sistema que permita la comunicación entre el sensor neuronal y una silla de ruedas eléctrica, utilizando la interfaz cerebro-ordenador, que es un panel que permite a un usuario comunicarse con una máquina, software o sistema (COPA-DATA, 2016), pudiendo aportar a la sociedad una herramienta que ayude a las personas con un alto grado de discapacidad motriz en su desenvolvimiento diario.

\section{MATERIALES Y MÉTODOS}

La investigación tecnológica planteada se desarrolló en la Universidad Nacional de Asunción Facultad Politécnica en el Grupo de Investigación en Electrónica Mecatrónica, la misma consistió en el diseño del sistema de control para el movimiento de una silla de ruedas eléctrica mediante señales neuronales empleando una interfaz cerebro computador. Los sensores del casco del Emotiv Epoc leen las señales eléctricas producidas por el cerebro y las envía inalámbricamente a un computador portátil. Las señales recibidas en el computador son procesadas por un software desarrollado con código abierto brindadas por Emotiv, que se encargan de interpretar y convertir en instrucciones, para luego enviarlas a un módulo electrónico ARDUINO que interviene en el control del movimiento de la silla de acuerdo con los gestos faciales o cognitivas realizados por el usuario. El esquema del sistema completo se muestra en la Figura 1. A continuación se detalla el funcionamiento de cada una de las partes del sistema.

\section{A-Adquisición de la señal}




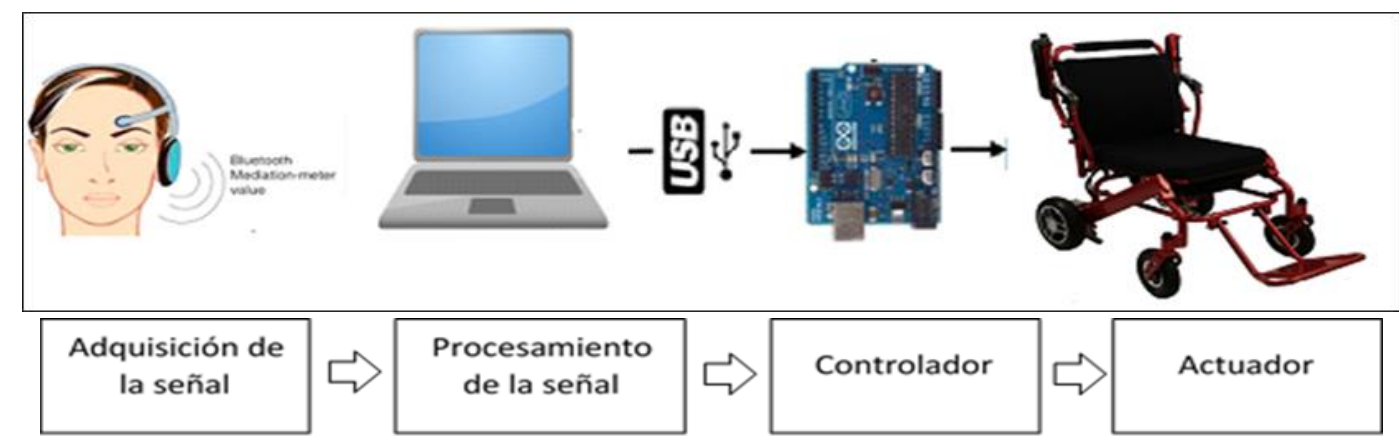

Figura 1. Esquema de control.

Desde la invención del EEG se han producido investigaciones que han estudiado la relación entre las ondas cerebrales y los diferentes estados de conciencia (Aznar Casanova, 2017), como también ha llevado al mismo a la creación de lectores de ondas neuronales como el Sensor Neuronal EPOC+ Emotiv, sensor designado para el desarrollo del proyecto, que por medio del cual se realizó la lectura del estado en el que se encuentre un usuario, o realizar el control de la silla eléctrica. Para la detección de la actividad cerebral mediante un sensor neuronal existen tipos de señales que se clasifican de acuerdo con el rango de frecuencia al que pertenecen, en la Figura 2 se puede observar a estas señales clasificadas dando lugar a los tipos de estados en el que se puede encontrar un usuario y que pueden ser detectados mediante un sensor neuronal.

\begin{tabular}{|c|c|c|c|}
\hline Ritmos & Frecuencias & Ondas & Estado \\
\hline Beta $(\beta)$ & $14-30 \mathrm{~Hz}$ & 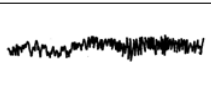 & $\begin{array}{ll}\dot{\text { - }} & \text { Enfoque } \\
\dot{\text { - }} & \text { Alerta } \\
\text { - } & \text { Ocupado } \\
\text { pensando }\end{array}$ \\
\hline Alfa $(\alpha)$ & $8-13 \mathrm{~Hz}$ & 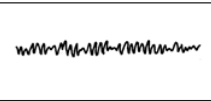 & $\begin{array}{ll} & \text { Relajado } \\
\text { - Imaginación } \\
\text { - Sueños } \\
\text { diurnos }\end{array}$ \\
\hline Tetha $(\theta)$ & $4-7 \mathrm{~Hz}$ & 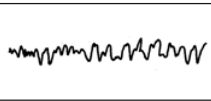 & $\begin{array}{ll}\text { - Meditación } \\
\text { - Flujo de ideas } \\
\text { - Enlace al } \\
\text { inconsciente }\end{array}$ \\
\hline Delta (ס) & $>3,5 \mathrm{~Hz}$ & 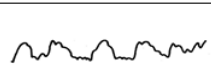 & $\begin{array}{ll}\text { - Inconsciencia } \\
\text { - Sueño } \\
\text { Profundo }\end{array}$ \\
\hline
\end{tabular}

Figura 2. Señales neuronales.

Los tipos de señales utilizados para el control de la silla de ruedas eléctricas fueron inicialmente los gestos faciales, que son las expresiones realizadas por el usuario. Las lecturas de estos tipos de movimientos realizados con el rostro fueron una sonrisa, un guiño, fruncir el ceño, etc.

Otro tipo de control utilizado para los movimientos de la silla de ruedas eléctrica fueron las señales cognitivas que son básicamente los pensamientos. Para la detección del Sensor Neuronal EPOC+Emotiv cuenta con un software de control de nombre "Emotiv Xavier Controlpanel" con el cual se puede obtener información del estado de conexión de cada uno de los 14 canales que posee el sensor, el nivel de batería con que cuenta, suites que permiten realizar entrenamientos tanto de gestos faciales como cognitivos, y una suite inercial ya que el sensor cuenta con un giroscopio, también es posible visualizar las señales de los diferentes estados en el que se podría encontrar un usuario (Emotiv, 2017). En la suite facial cuenta con un avatar que imita los gestos que el usuario realiza y a su vez con opciones que permiten ajustar la sensibilidad de detección de algunos gestos. En la suite cognitiva se cuenta con un cubo 3D virtual para mostrar una representación animada de la detección (Soler, Marco, Rodríguez, Harold, Peña \& César, 2014), que permite "observar" el tipo de movimiento que desea realizar un usuario tan solo pensándolo, refiriéndose a esos con los movimientos del como subir, bajar, empujar, estirar, mover hacia un lado u otro el cubo.

Luego se tiene la suite Inercial Sensor, en la cual aparte de visualizar el estado de conexión de los canales del sensor neuronal, es posible también ver el movimiento de un cursor siguiendo los movimientos que realice un usuario con la cabeza, esto sea mover la cabeza para la derecha, izquierda, arriba o abajo.

\section{B-Procesamiento de la señal}

Una vez obtenidas las señales mediante el sensor neuronal, son enviadas al computador por comunicación serial mediante Bluetooth. Estas son procesadas con la ayuda de tres softwares comprendidos por el Emotiv Control 
Panel, Emokey, EmoEngine y Putty, que respectivamente realizan la correcta detección y clasificación de las señales por parte el Emotiv Control Panel para posteriormente ser enviadas al Emokey, que permite conectar los resultados de detección recibidos y este traducirlas a caracteres bajo reglas establecidas por el usuario y por último los envía al Software Putty, que es un cliente SSH y telnet (Putty, 2019), quien se encarga del envió y la comunicación serial con la placa de control Arduino de la interfaz electrónica.

\section{C- Interfaz electrónica}

La placa de control se encarga de la recepción de la señal. La placa de control se encarga de la recepción de la señal obtenida y procesada por el sensor y a su vez envía las órdenes a los circuitos de potencia para la activación de los motores de la silla. La placa de control utilizada fue la Arduino Mega 2560 (Arduino, 2020). Los circuitos de potencia se encuentran conformados por un Puente $\mathrm{H}$ y una Bancada de relés quienes respectivamente se encargan de aplicar el PWM y el sentido de giro a las ruedas de las sillas, permitiendo así a la misma poder realizar las siguientes acciones: avance, retroceso, giro a la derecha, giro a la izquierda y parar. La lógica de control de la silla de ruedas se basa en el diagrama de flujo representado en la Figura 3, donde se tiene que la interfaz electrónica recibe un carácter, interpreta éste como una orden de control y a partir del mismo identifica que tipo de acción debe realizar, en qué estado se encuentra la silla de ruedas (en movimiento o frenada) y de acuerdo a ello acciona o frena y posteriormente realiza la acción, permitiendo así tener un ciclo continuo y bajo protección. También cuenta con un control de lazo cerrado PID el cual nos permite tener un mayor control sobre el comportamiento de la silla al momento de llevar a cabo una acción, ya que la silla de ruedas trata de un robot móvil de tracción diferencial, el control PID nos permite corregir la diferencia de velocidad en ambas ruedas y así tener un mejor control y lograr el comportamiento deseado en la silla de ruedas

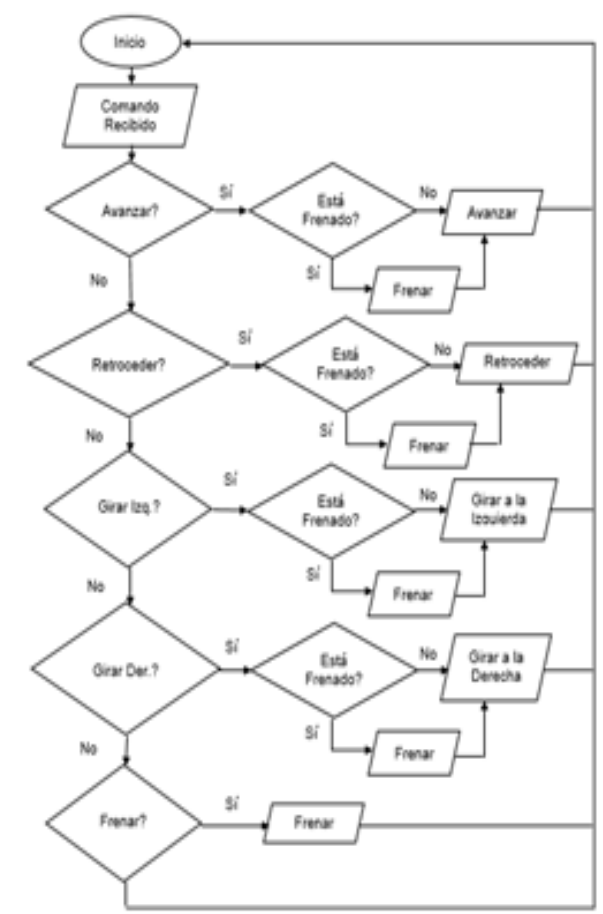

Figura 3. Diagrama de flujo del programa de control del Arduino Mega.

1-Circuitos de potencia de la silla de ruedas: El conjunto de circuitos encargados del accionamiento de la silla de ruedas se encuentra constituido por una combinación de una bancada de relés y un puente $\mathrm{H}$ Arduino IBT-2, que es un controlador de motor económico y de alta potencia basado en dos chips BTS7960 (Hessmer, 2013), quienes se encargan de realizar respectivamente la conmutación de giros y la aplicación correspondiente del rpm deseado. El puente $\mathrm{h}$ recibe los comandos de rpm y los aplica a cada rueda, y a su vez recibe los comandos de giro para pasar a la bancada de relés, quien se encarga de la conmutación de giros de cada rueda, permitiendo que estas giren en el mismo sentido o en sentidos opuestos.

2-Módulo conversor de voltaje: $\mathrm{El}$ módulo convertidor LM2596 es una fuente de alimentación conmutada (Electronilab, 2020), fue utilizado para la alimentación de los distintos bloques de electrónica que componen el tablero de control, ajustando así de $24 \mathrm{~V}$ a 12 $\mathrm{V}$ para la alimentación de los encoders y de 24 $\mathrm{V}$ a $5 \mathrm{~V}$ para las resistencias pull-up de estos.

\section{D- Silla de rueda eléctrica}

La silla utilizada, fue facilitada por el laboratorio del Grupo de Investigación de Electrónica y Mecatrónica (GIEM), la cual se encuentra ensamblada y cuenta con motores 
DC Brushed, circuitos acopladores para el manejo de los sentidos de dirección y velocidad de este. También cuenta con encoders, los cuáles son sensores que generan señales digitales en respuesta al movimiento (Barcojo, 2014), éstos permiten obtener las revoluciones por minuto de las ruedas y así tener un mayor control del vehículo. Las ruedas de tracción son las ruedas traseras ya que en ellas están ubicados los motores DC, las ruedas delanteras actúan como guía del vehículo, ya sea para un trayecto rectilíneo o realizando un giro. El tipo de silla es del tipo robot o vehículo móvil con locomoción por ruedas, con configuración de tracción diferencial, que se caracteriza porque el movimiento se consigue con dos ruedas, acopladas cada una a su propio motor, teniendo normalmente una o dos ruedas de apoyo, en este caso se trata de una silla con dos ruedas de apoyo. De esta forma se logra que el robot avance en línea recta fijando las revoluciones de ambos motores a la misma velocidad, teniendo también la posibilidad de hacerla girar en una u otra dirección, permitiendo que gire sobre su propio eje cuando las velocidades tienen igual magnitud, pero sentidos opuestos.

Con el prototipo de silla de ruedas eléctrica se realizaron pruebas de funcionamiento del interfaz de control, el cual se ejecutó por dos métodos totalmente independientes: control facial y el cognitivo.

\section{A-Método de control facial.}

Para las pruebas de funcionamiento se contó con la colaboración de tres personas de sexos tanto masculino como femenino con edades entre 25 a 27 años, en dichas pruebas se realizaron el control del movimiento del prototipo de la silla de ruedas eléctrica mediante expresiones faciales.

En la Tabla 1 se muestra la asignación de gestos a los tipos de movimiento que debe realizar la silla en el modo expresivo.

\section{B- Método de control cognitivo}

Las pruebas fueron realizadas por tres voluntarios de ambos sexos, masculino y femenino, con un rango de edad 27 a 32 años, quienes llevaron a cabo el siguiente procedimiento.

Tabla 1. Asignación de gestos en el modo expresivo.

\begin{tabular}{l|l}
\hline \multicolumn{1}{c|}{ Acción } & \multicolumn{1}{c}{ Gesto } \\
\hline Ir hacia adelante & Sonreír \\
Ir hacia la derecha & Guiño del ojo derecho \\
Ir hacia la izquierda & Guiño del ojo izquierdo \\
Ir hacia atrás & Fruncir el ceño \\
Frenar & Parpadeo \\
\hline
\end{tabular}

Primero un entrenamiento previo del Control Cognitivo con ayuda de la suite cognitiva del software Emotiv Xavier Control Panel. En esta suite cognitiva se cuenta con un cubo 3D que te permite "observar" el tipo de movimiento que desea realizar un usuario tan solo pensándolo, refiriéndose a esos con los movimientos del como subir, bajar, empujar, estirar, mover hacia un lado u otro el cubo, en la Figura 4 se puede apreciar cómo es la suite cognitiva.

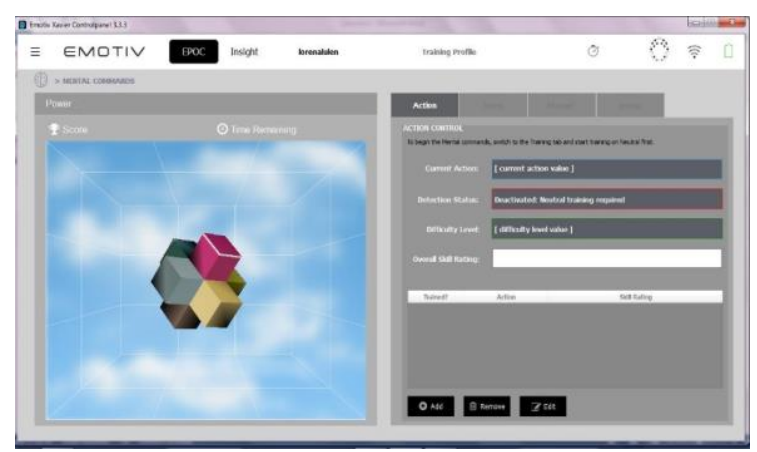

Figura 4. Suite Cognitiva del Emotiv Xavier Control Panel.

Segundo, el control del movimiento del prototipo de la silla de ruedas eléctrica mediante el control de modo cognitivo. Al igual que en el control con gestos faciales (véase Tabla 1), en el control de modo cognitivo también se realizó la asignación de pensamientos para cada tipo de movimiento que debe realizar la silla, en la Tabla 2 se especifican las asignaciones.

Tabla 2. Asignación de pensamientos en el modo cognitivo.

\begin{tabular}{l|c}
\multicolumn{1}{c|}{ Acción } & Gesto \\
\hline Ir hacia adelante & Push \\
Ir hacia la derecha & Righ \\
Ir hacia la izquierda & Left \\
Ir hacia atrás & Pull \\
Frenar & Neutral \\
\hline
\end{tabular}




\section{RESULTADOS}

El porcentaje de reconocimiento de los gestos faciales obtenidos durante los entrenamientos realizados por parte de los tres voluntarios, para el movimiento de silla de ruedas eléctrica, se muestra en la Tabla 3.

Tabla 3. Porcentaje de reconocimiento de los gestos por parte de cada voluntario.

\begin{tabular}{c|c|c|c|c|c}
\hline Persona & Sonreír & $\begin{array}{c}\text { Guiño } \\
\text { izquierdo }\end{array}$ & $\begin{array}{c}\text { Guiño } \\
\text { derecho }\end{array}$ & $\begin{array}{c}\text { Fruncir } \\
\text { el ceño }\end{array}$ & Parpadeo \\
\hline Femenino & 100 & 35 & 30 & 55 & 95 \\
Masculino & 100 & 50 & 35 & 60 & 100 \\
Masculino & 100 & 45 & 40 & 50 & 90 \\
\hline
\end{tabular}

En la Tabla 4 se indican los tiempos de entrenamiento del Control Cognitivo con ayuda de la suite cognitiva del software Emotiv Xavier Control Panel que le tomo a cada voluntario, observando que para cada uno varía el tiempo de entrenamiento de acuerdo con el estado de concentración en el que se encuentre.

Tabla 4. Tiempo de entrenamiento que le llevó a cada voluntario.

\begin{tabular}{l|c}
\multicolumn{1}{c|}{ Poluntario. } & Tiempo de entrenamiento \\
\hline Femenino & 90 minutos \\
Masculino & 75 minutos \\
Masculino & 45 minutos \\
\hline
\end{tabular}

Y por último en la Tabla 5 se muestran los porcentajes obtenidos, durante los entrenamientos, para cada tipo de pensamiento en cada voluntario en la cual se refleja el nivel de entrenamiento que fue adquiriendo el usuario.

Tabla 5. Porcentaje de reconocimiento de los pensamientos durante los entrenamientos.

\begin{tabular}{c|c|c|c|c|c}
\multicolumn{6}{c}{ durante los entrenamientos. } \\
\hline Persona & Pull & Push & Righ & Left & Neutral \\
\hline Femenino & 94 & 65 & 54 & 53 & 100 \\
Masculino & 75 & 68 & 66 & 66 & 100 \\
Masculino & 66 & 75 & 75 & 70 & 100 \\
\hline
\end{tabular}

Con dos voluntarios con discapacidad motriz se realizó como prueba final, obteniendo una eficiencia de $75 \%$ para los estímulos de modo expresivo y $65 \%$ para los cognitivos para el control de la silla.

\section{DISCUSIÓN}

Se realizó la comparativa con un proyecto similar realizado en la Facultad de Ingeniería Eléctrica y Computación de la Universidad Estatal de Campinas, Brasil, que utiliza la tecnología RealSense de Intel (Observatorio Tecnológico de Hidalgo, 2016) para realizar el control de una silla de ruedas mediante control facial, realizando gestos, y éstos a la vez, son captados por una cámara de video e interpretados por un computador utilizando reconocimiento facial. Este proceso de captación de gestos faciales es mucho más lento que la captación de señales neuronales ya que el tiempo de respuesta de la cámara más el procesado de la señal hace que tome mucho tiempo realizar la acción requerida. Además, las cámaras con tecnología RealSense de Intel puede ser costosas, lo que hace a la utilización de las herramientas Emotiv más atractivas a la hora de elegir un medio para realizar el movimiento de una silla de ruedas eléctrica. A su vez, este proyecto introduce nuevos elementos, que lo distinguen de publicaciones anteriores. (Guin \& Bikash Baishya, 2013) presentó un prototipo de silla de ruedas controlada mediante electrodos, sin embargo, el trabajo se centra en analizar la calidad de las señales captadas por estos electrodos. Una publicación posterior, (Sinha \& Kanthi, 2016) informa del uso del Neurosky Mindwave Headset para controlar una silla de ruedas con un GUI desarrollado en Java. También se ha utilizado un EEG sistema para controlar una silla de ruedas utilizando el eye blink para moverla en cuatro direcciones. Pantech Solutions (2017) para detectar las ondas cerebrales de atención y parpadeo del ojo, ha empleado un Neurosky Headset, que se comunica vía bluetooth con una aplicación en Android; mientras que en esta investigación el sistema de control está desarrollado en un microcontrolador Arduino que se encarga del movimiento de la silla, permitiendo el control y 
manejo efectivo de una silla de ruedas utilizando una interfaz cerebro-computadora que interpreta las señales cerebrales al momento de realizar diferentes gestos faciales.

El protocolo elaborado para la evaluación garantizó la realización adecuada de cada una de las tareas por el usuario. Se puede obtener una efectividad mayor con un entrenamiento previo, lo que permitiría la adaptación a los distintos movimientos de la silla de ruedas. Los datos obtenidos muestran que el sistema respondió apropiadamente a las acciones requeridas para la movilidad de la silla de ruedas.

\section{CONCLUSIONES}

De acuerdo con el estudio realizado a lo largo del proyecto, se pudo concluir que hemos obtenido los resultados esperados, la comunicación del sensor neuronal Emotiv y la placa de desarrollo Arduino obteniendo como el accionamiento de la silla. Observando a su vez que para cada usuario el casco debe ser "entrenado" por el mismo y de esa forma el sistema ir adecuándose al usuario hasta conseguir el nivel de respuesta deseado.

El logro de este tipo de proyectos nos permitirá la evolución del mismo pudiendo optimizarlo y agregando una opción más de control a la misma, y así poder aportar a la sociedad una herramienta que ayude al desplazamiento de personas con un alto grado de discapacidad motriz.

\section{AGRADECIMIENTOS}

Agradecimiento a los miembros del Grupo de Investigación en Electrónica y Mecatrónica por las orientaciones y asesoría técnica, así como al Departamento de Investigación y Postgrado por el apoyo ofrecido para el desarrollo de este trabajo.

\section{FINANCIACIÓN}

La investigación fue presentada por la Facultad Politécnica y financiado con fondos de investigación del Rectorado de la Universidad Nacional de Asunción, Paraguay. Convocatoria 2016.

\section{REFERENCIAS BIBLIOGRÁFICAS}

Ang, K. K., Ang, K.K., Guan, C., Chua, K.S., Ang, B.T., Kuah, C.W...Zhang, H. (2011, Octubre). A Large Clinical Study on the Ability of Stroke Patients to Use an EEG-based Motor Imagery Brain-Computer Interface. Clinical EEG and Neuroscience, 42(4).

Arduino (2020). Disponible en: https://www.arduino.cc/en/Main/ArduinoBoardMe ga 2560

Aznar Casanova, J. A. (2017). La consciencia: La interfaz polinómica de la subjetividad http://www.ub.edu/pa1/node/130

Barcojo, A. R. (2014). Unidad Didáctica: Control y robótica. Sección: Sensores. http://cmapspublic2.ihmc.us/rid=1H2F1807LJP0SG2-J1J/encoder.pdf

COPA-DATA. (2016). Interfaz Hombre-Maquina (HMI).

Disponible en: https://www.copadata.com/es/soluci ones-hmi-scada/interfaz-hombre-maquina-hmi/

Electronilab. (2020). Módulo LM2596 Convertidor de Voltaje DC-DC Buck 1.25V-35V. Disponible en https://electronilab.co/tienda/modulo-lm2596convertidor-de-voltaje-dc-dc-buck-1-25v-35v/

Emotiv. (2017). Emotiv Software Development Kit.cmap.polytechnique.fr/ jingrebeccali/ecole_det e_france_excellence_files_2017/UserManualForE motivXavierControlPanel_Epoc.pdf

Guin, A. \& Bikash Baishya, B. (2013) Brain Controlled Wheelchair using LabVIEW. Disponible en: https://www.pantechsolutions.net/blog/wpcontent/uploads/2017/10/Brain-controlled-wheelchair-using-Labview.pdf

Hessmer, D. R. (2013). IBT-2 H-Bridge with Arduino. Disponible en: http://www.hessmer.org/blog/2013/12/28/ibt-2-hbridge-with-arduino/

National Spinal Cord Injury Statistical Center. (2011). Spinal Cord Injury Facts and Figures at a Glance. Birmingham, Estados Unidos: Universidad de Alabama, NSCISC.

Observatorio Tecnológico de Hidalgo. (2016). Esta silla de ruedas se controla con expresiones faciales. Disponible en: https://otech.uaeh.edu.mx/noti/index.php/tech/estasilla-de-ruedas-se-controla-con-expresionesfaciales/

Pantech Solutions. (2017) Brain controlled wheel chair [Internet]. Chennai: Pantech solutions. Disponible en: https://www.pantechsolutions.net/braincontrolled-wheel-chair

Putty.(2019) Disponible en: https://www.putty.org/ 
Sinha, U. \& Kanthi, M. (2016) Mind Controlled Wheelchair. IJCTA, 9(39): 19-28. Disponible en: https://www.pantechsolutions.net/bl og/wp-content/uploads/2017/10/Brain-controlledwheel-chair.pdf

Soler V., Marco A., Rodríguez A., Harold A., Peña C. \& César A. (2014). Desarrollo de un robot explorador operado mediante neuroseñales. Revista Politécnica,10(19), 125-134. Disponible en: http://revistas.elpoli.edu.co/index.php/pol/article/do wnload/479/507
Wang, S., Esfahani, E.T. \& Sundararajan, V. (2012). Evaluation of SSVEP as Passive Feedback for Improving the Performance of Brain Machine Interfaces. ASME 2012 International Design Engineering Technical Conferences \& Computers

World Health Organization, The World Bank. (2011) World report on disability. Ginebra, Suiza : OPS 